\title{
Theoretical study of heat transfer processes in Heusler-type magnetic microwires
}

\author{
O. O. Pavlukhina ${ }^{\dagger, 1}$, V. V. Sokolovskiy ${ }^{1,2}$, V. D. Buchelnikov ${ }^{1,2}$, M. A. Zagrebin ${ }^{1,2}$ \\ †pavluhinaoo@mail.ru
}

\author{
${ }^{1}$ Chelyabinsk State University, 129 Bratiev Kashirinykh St., Chelyabinsk, 454001, Russia \\ ${ }^{2}$ National University of Science and Technology “MISiS”, 4 Leninskiy Av., Moscow, 119991, Russia
}

\begin{abstract}
In the present work, two types of geometrical arrangement (C-1 and C-2) of Heusler-type $\mathrm{Ni}_{45} \mathrm{Co}_{5} \mathrm{Mn}_{37} \mathrm{In}_{13}$ microwires in a cooling cell are investigated theoretically. The influence of the location of microwires on the course of heat transfer processes was investigated. The diameter of microwires was ranged from 10 to $50 \mu \mathrm{m}$, while the length of microwires was fixed to $1 \mathrm{~mm}$. The calculations were carried out for two cells with differently arranged layers of microwires. The volume of the cooling cell and the cell parameters were similar for both cells. To simulate the heat transfer process in a three-dimensional cooling cell with microwires, we considered a mathematical model including the heat conduction and convection mechanisms, as well as the coolant motion. To model heat transfer processes, the system of Navier-Stokes, continuity and thermal conductivity equations is solved by the finite-element method. It is shown that the relaxation time is about $2.4 \mathrm{~ms}$ and $2.2 \mathrm{~ms}$ for cooling cells C-1 and C-2, which contain microwires with a diameter of $50 \mu \mathrm{m}$, and about $0.9 \mathrm{~ms}$ and $0.8 \mathrm{~ms}$ for cooling cells C-1 and $\mathrm{C}-2$, which contain microwires with a diameter of $10 \mu \mathrm{m}$. A lower relaxation time indicates that heat transfer can occur faster in the C-2 cell with the same initial coolant velocity and equal wire thickness. It is also established that the transfer of thermal energy occurs more efficiently and faster in the cell C-2. Our study reveals that the geometrical arrangement of C-2 cell is more optimal.
\end{abstract}

Keywords: Heusler alloys, magnetic wires, heat transfer, simulation.

\section{Introduction}

The magnetocaloric effect (MCE) is an isothermal change in entropy $\left(\Delta S_{i s o}\right)$ and an adiabatic change in temperaturme $\left(\Delta T_{a d}\right)$ in a magnetic solid in response to a variation in the magnetic field. The opportunity to apply MCE in new refrigeration appliances is a sufficient factor to stimulate theoretical and experimental studies in the field of solid-state magnetic cooling. The huge interest is confirmed by at least several major factors. Nowadays, there are many effective thermodynamic cycles and developed prototypical devices based on the solid-state magnetic cooling technology $[1,2]$.

The search of new magnetocaloric alloys, which are stable, cheap, and non-toxic, is of interest to many research groups. At present, there are several families of magnetocaloric materials, including $\mathrm{Mn}-\mathrm{Ga}-(\mathrm{C}, \mathrm{N})$, Gd-Si-Ge, Fe-Rh, La-Fe(Si, Al), Ni-Mn-(Ga, In, Sn, Sb) and $\mathrm{Mn}-\mathrm{Fe}-(\mathrm{P}, \mathrm{As})$, which have perspectives in the solid-state cooling technology [3-8]. Ni-Mn-based alloys of the Heusler family have attracted a lot of interest due to several unique properties associated with martensitic transformation, such as thermally- and magnetic-induced shape memory, giant magnetostrains and magnetoresistance, conventional and inverse MCE and others [7-11]. Note that with the addition of the fourth element, the temperatures of both martensitic and magnetic transition become sensitive. One of the interesting transition elements is cobalt. A small amount of Co in Ni-Mn-(Ga, In, Sn, Sb) can decrease greatly the temperature of the martensitic transition and increase the Curie temperature of austenite [12]. Moreover, Liu et al. [3] and Gottschall et al. [13] reported record values of $\mathrm{MCE}$ $\left(\Delta T_{a d} \approx-6\right.$ and $\left.8 \mathrm{~K}\right)$ in $\mathrm{Ni}_{1.8} \mathrm{Co}_{0.2} \mathrm{Mn}_{1.48} \mathrm{In}_{0.52}$ with a change in the magnetic field up to $1.9 \mathrm{~T}$. However, these values of $\Delta T_{a d}$ are observed for the first cycle only, while subsequent values are sufficiently smaller. A drastic change in MCE is caused by a large thermal hysteresis of the martensitic transition.

On the other hand, the solid-state refrigeration contains problems related to the size of the refrigerant and the geometry of the cooling cell. Evidently, in order to achieve the efficient heat transfer, the ratio between the surface area and the volume of the working body should be as high as possible. Therefore, the coolant can quickly remove heat from the cooling material. Thus, ribbons, thin plates, meshes, and wires [14-26] are promising for the magnetic cooling technology. Khovaylo et al. [20] reviewed the problems of using ribbons, wires, and thin films in magnetic refrigeration. In particular, the magnetic properties of size-reduced samples differ usually from the bulk ones. In addition, the estimation of magnetic and magnetocaloric properties of size-reduced samples includes technical problems.

Recent experimental findings have shown that the list of candidates for refrigerant includes magnetic microwires of the Heusler-type [27-31]. For instance, Zhukova et al. [21] reported on the increase in the Curie temperature from 200 to $270 \mathrm{~K}$ for $\mathrm{Ni}_{50} \mathrm{Mn}_{32} \mathrm{In}_{15}$ glass-coated microwires after addition of Co about 5 at.\%. Besides, annealing of Ni-Co-Mn-In 
microwires leads also to growing of the Curie temperature. As regards the design of the cooling cell, Balli et al. [17] reviewed a series of analytical and numerical studies on the flow field distributions. Nielsen et al. [25] and Engelbrecht et al. [26] performed a numerical modeling of the heat transfer process with account of parasitic heat losses for a set of parallel plates. Sarlah et al. [22] remarked that a promising direction for the magnetic cooling cell is parallel plates of MCE material.

Evidently, the choice of the geometry for the regenerator or magnetic cooling cell is an important problem in the magnetic cooling technology. There were reports about engineering design and numerical investigations of active magnetic regenerators, including meshes of channels with square cross-sections, packed particles, and stacks of parallel plates of magnetocaloric materials. To our knowledge, theoretical investigations of thermophysical properties of Heusler microwires have not been reported previously, except for Refs. [31,32]. Therefore, we focus attention on the three-dimensional distribution of heat transfer and the thermophysical properties of the Heusler-type $\mathrm{Ni}_{45} \mathrm{Co}_{5} \mathrm{Mn}_{37} \mathrm{In}_{13}$ microwires. In this paper, we investigate two types of wire arrangement in magnetic cooling cells, as well as the influence of this arrangement on heat transfer processes.

\section{Computational details}

To simulate the heat transfer process in a three-dimensional cooling cell with microwires, we consider a mathematical model including the heat conduction and convection mechanisms, as well as the coolant motion. In such a manner, the system of Navier-Stokes, together with continuity and thermal conduction equations for the fluid and solid is given by

$$
\left\{\begin{array}{l}
\operatorname{div} \mathbf{u}=0, \\
\rho \frac{\partial \mathbf{u}}{\partial t}+\rho(\mathbf{u} \cdot \nabla) \mathbf{u}=\eta \Delta \mathbf{u}, \\
\rho C_{p(l)} \frac{\partial T}{\partial t}+\nabla\left(-k_{l} \nabla T\right)=\nabla\left(-\rho C_{p(l)} \mathbf{u} T\right), \\
\rho_{1} C_{p(s)} \frac{\partial T}{\partial t}+\nabla\left(-k_{s} \nabla T\right)=0 .
\end{array}\right.
$$

where $T=T(x, y, z)$ is the temperature, $C_{p(l)}$ and $C_{p(s)}$ are the heat capacities of the liquid $(l)$ and solid $(s), k_{l}$ and $k_{s}$ are the corresponding thermal conductivities, $\eta$ is the viscosity, $\rho$ is the density, $\mathbf{u}=\mathbf{u}(x, y, z, t)$ is the velocity vector of the fluid at the point $(x, y, z)$ in space at time $t$.

To solve the system of equations numerically with account of boundary conditions, the finite element method was implemented $[33,34]$. The following assumptions were proposed to describe the boundary conditions. On the one hand, a number of factors such as a viscosity, coolant velocity, surface roughness of a solid material, as well as its shape, can strongly affect the coolant flow leading to a change in its velocity. On the other hand, the coolant flow can be delayed by the solid wall of the cell due to the appearance of molecular cohesion forces between them. Moreover, the boundary conditions for the motion equation of a viscous fluid require vanishing the fluid flow velocity when the fluid is in contact with a rigid solid surface. Therefore, the constant incoming coolant velocity was proposed and the heat flux inflowing/outflowing in a mesh element was assumed to be constant. Besides, the cell walls were fully insulated. In addition, the knowledge of the temperature difference $\left(\Delta T_{a d}\right)$, which is realized in the material at a critical temperature whereby applying a magnetic field adiabatically, is sufficient because the cooling cell involves a magnetocaloric material. Thus, the initial temperature for both the coolant and the microwires was equal to the martensitic transition temperature $\left(T_{m}\right)$ for $\mathrm{Ni}_{45} \mathrm{Co}_{5} \mathrm{Mn}_{37} \mathrm{In}_{13}$. The constant coolant inlet velocity $\left(U=2.5 \mathrm{~ms}^{-1}\right)$ was taken at a constant temperature, defined as $T_{m}+\Delta T_{a d}$, where $T_{m} \sim 320 \mathrm{~K}$, and $\Delta T_{a d} \sim-6 \mathrm{~K} \mathrm{[3]}$.

The choice of the cooling cell geometry was governed by the experimental findings. Realistic cooling cells should contain a certain amount of microwires arranged in several layers. Therefore, to optimize the magnetic cooling cell, it is necessary to investigate the arrangement of the wires relative to each other in a cell consisting of a large number of wires. Thus, two possible arrangements of microwires denoted as C-1 and C-2 were considered (See Fig. 1). There is a space between the wires filled with the coolant for heat transfer. In the first and second cases, the wires were squarely and hexagonally packed, respectively. Moreover, equal volumes and cell parameters of the cooling cell for both C-1 and C-2 systems were taken into consideration.

The geometry of the cell was as follows: $0.5 \mathrm{~mm}$ (height) $\times$ $\times 0.4 \mathrm{~mm}$ (width) $\times 2.5 \mathrm{~mm}$ (length). The wire length was fixed to $1 \mathrm{~mm}$, while its width ranged between 10 and $50 \mu \mathrm{m}$. In the course of our simulations, we increased the diameter of the microwires keeping their initial position. To perform numerical simulations, the mesh for the cooling cell, divided into a collection of finite elements, was constructed. Note that each element was represented by a set of differential equations. Moreover, the number of mesh elements depended on the wire width.

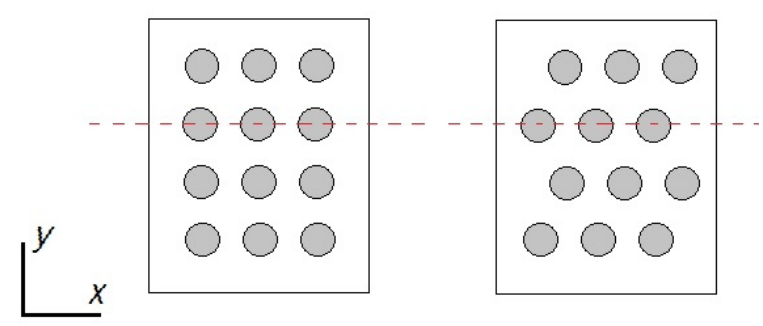

Fig. 1. The three-dimensional model of C-1 and C-2 cooling cells, which are projected onto the $x y$ plane. Circles denote wire sections.

\section{Results and discussion}

In this section, we discuss our results of heat transfer simulations in the cooling cell consisting of the Heusler-type Ni-Co-Mn-In microwires. The model parameters for $\mathrm{Ni}_{45} \mathrm{Co}_{5} \mathrm{Mn}_{37} \mathrm{In}_{13}$ microwires and coolant (water) were taken by analogy with Refs. $[3,10,11]$. Note that we consider the composition, which is similar to $\mathrm{Ni}_{45.2} \mathrm{Mn}_{36.7} \operatorname{In}_{13} \mathrm{Co}_{5.1}$ [3]. According to experimental work [3], the $\mathrm{Ni}_{45.2} \mathrm{Mn}_{36.7} \mathrm{In}_{13} \mathrm{Co}_{5.1}$ composition possesses the following martensitic temperatures: $A_{s}=317 \mathrm{~K}, A_{f}=327 \mathrm{~K}$ and $M_{s}=319 \mathrm{~K}$, $M_{f}=311 \mathrm{~K}$. 
The temperature difference $T_{m}-\Delta T_{a d}(314 \mathrm{~K})$ is chosen as the initial temperature for both the coolant and the microwires. It is considered that the coolant flow is fed into the system at a speed of $U(2.5 \mathrm{~m} / \mathrm{s})$ and temperature $(320 \mathrm{~K})$. Before discussing the results obtained, it is necessary to define the thermal control points positioned on the microwires. These points for both systems under consideration are presented in Fig. 2. The dash line (Fig. 1) indicates the considered plane $x z$. Due to the fact that the thermal relaxation time of microwires up to the coolant temperature is high, the following criterion is assumed to estimate the relaxation time: the microwire is heated when the temperature difference between the coolant and the microwire decreases in $e$ times (i.e. up to $317.79 \mathrm{~K}$ ). In order to study the relaxation time and to account the influence of all neighboring microwires on the heat transfer process, we choose a microwire centrally located for both types of the cooling cell. In regard to real systems, a similar cooling cell must be translated many times. Nevertheless, all microwires will be surrounded in a real cell by analogy with the considered one. Therefore, the theoretical modeling considered here allows us to evaluate temperature relaxation data close to the data for a real system.

Fig. $3 \mathrm{a}, \mathrm{b}$ illustrate the thermal relaxation time between the coolant and the microwires with $50 \mu \mathrm{m}$ in both $\mathrm{C}-1$ and C-2 cells. Temperature curves are measured from the corresponding thermocouples (see points in Fig. 2). Here the results with the corresponding symbols are shown for three control points presented in Fig. 2. It can be seen from both figures that the optimal temperature control point is a thermocouple located at the final part of the middle microwire and shown by the rhombus symbol. This is due to the fact that at this point the heating is slower (Fig. $3 \mathrm{a}, \mathrm{b}$ ). This suggests that the heating process has passed for all the wires in the system.
Fig. 4 shows the calculation results of the thermal relaxation time, which are taken from the optimal control point located at the final part of the middle microwire. Here the results are presented for both types of cooling cells, including microwires with a diameter of 20 and $50 \mu \mathrm{m}$. As we already mentioned above, the relaxation time is estimated at a temperature of $317.79 \mathrm{~K}$. Thus, in the case of wires with a diameter of $50 \mu \mathrm{m}$, the relaxation time is about 2.2 and $2.4 \mathrm{~ms}$ for C-2 and C-1 cell, respectively. As a result, we can conclude that the arrangement of microwires in the C-2 cell is optimal due to the less relaxation time, suggesting that the heat transfer occurs faster in C-2 cell at the equal coolant velocity and diameter of microwires. Besides, it can be associated with different canal widths between the wires in the cell. For both C- 1 and C- 2 cells, the $x$-axis distance along between the nearest wires is equal, while the $y$-axis distance for $\mathrm{C}$ - 2 cell is larger because of the layer shift of microwires. We would like to remind that $\mathrm{C}-1$ and $\mathrm{C}-2$ cells have different geometry of the arrangement of microwires. But the three-dimensional parameters of the considered cells are equal.

In order to estimate the velocity of the heat transfer fluid for C-1 and C-2 cooling cells, we further analyze the velocity distributions for both cells, which are illustrated in Fig. 5. Here, the contour maps of the velocity distributions are shown in the $x y$ plane, which intersects the $z$-axis at the point of the third control point (see rhombic symbol in Fig. 2). It important to note that in order to estimate the velocity of the heat transfer fluid, the central area of the cell is worth considering. In this case, the central microwires are surrounded by others. In real systems, a similar cell will be translated many times in the $x$ and $y$ directions, so all the wires in such a cell will be located in an environment similar to the wire closer to the center of the cell. One can see from Fig. 5, the velocity of heat transfer fluid between neighbor

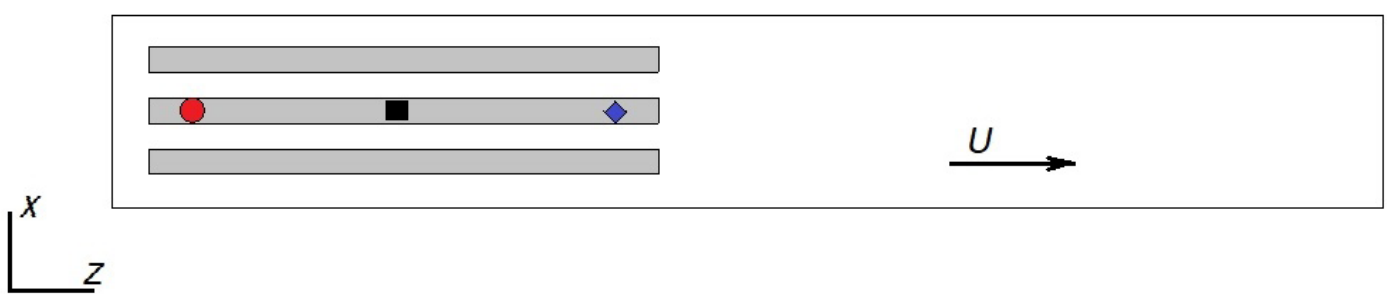

Fig. 2. Three control points (thermocouples) located at the begin, middle, and final part of the middle wire.

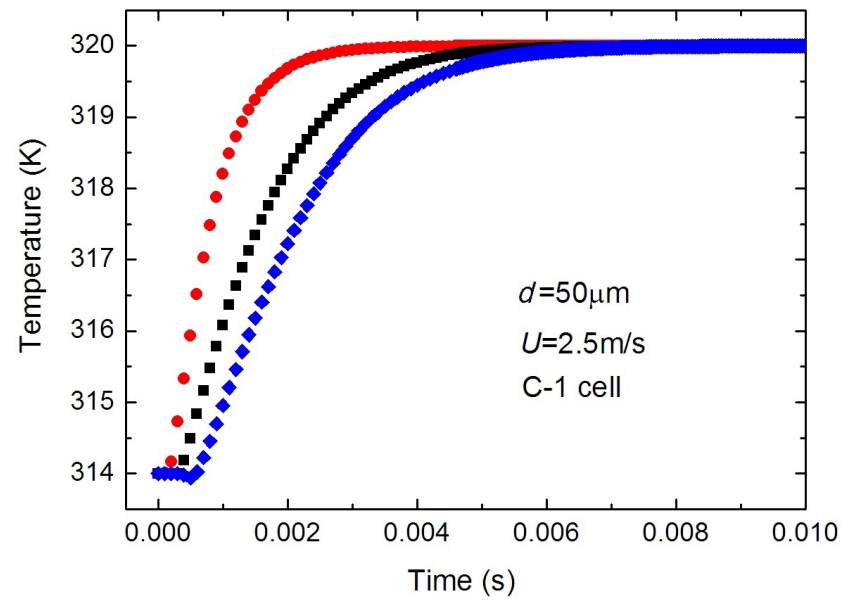

a

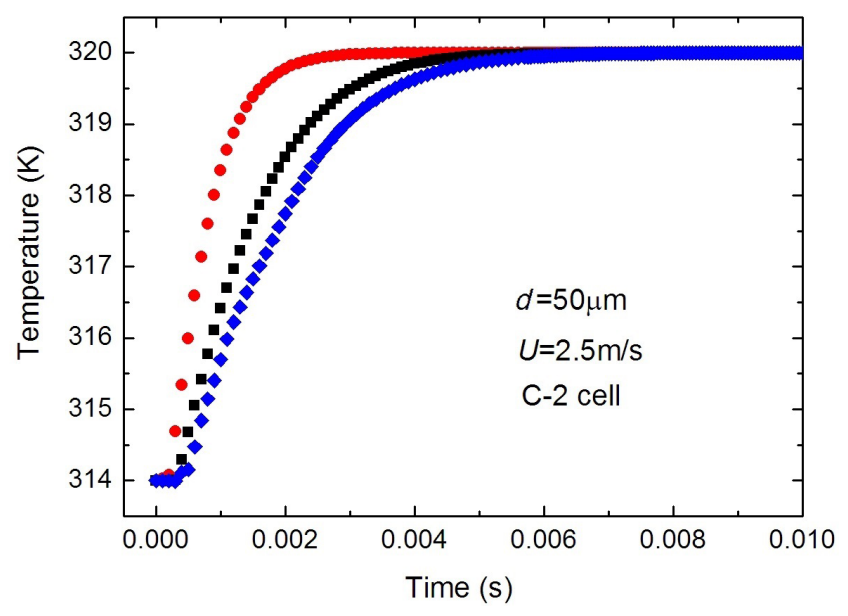

$\mathrm{b}$

Fig. 3. (Color online) The temperature difference between the coolant and microwires as a function of time for C-1 (a) and C-2 cooling cells (b). 


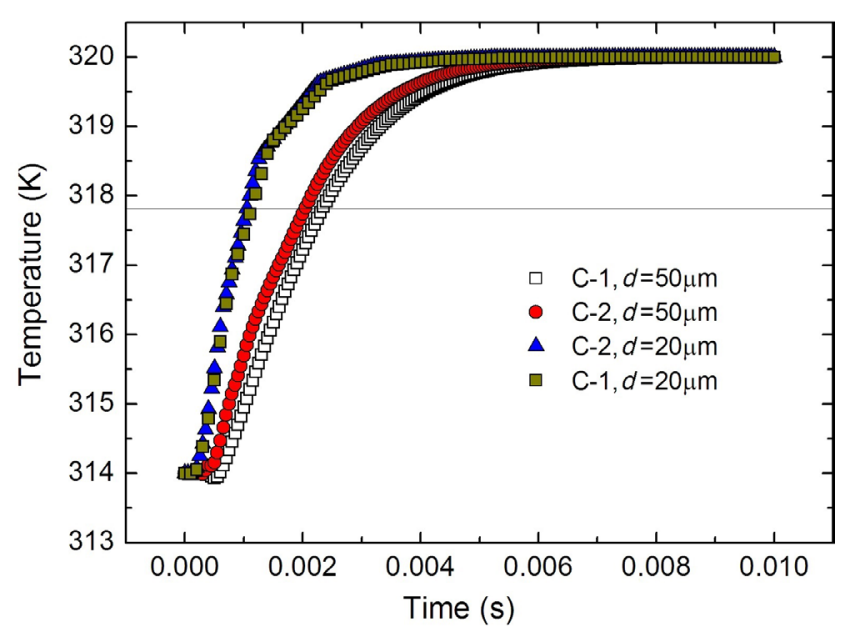

Fig.4. (Color online) The temperature difference between the coolant and the microwires as a function of time for C-1 and C-2 cooling cells involving microwires with a diameter of 20 and $50 \mu \mathrm{m}$. Data are taken from the thermocouple located at the final part of the middle microwire. The temperature corresponding to the relaxation time is marked with a horizontal line.

mircrowires is higher for C-2 cell. Our simulations reveal also that the coolant velocity in the system is nonuniform due to the difference between canal widths. The latter plays an important role in the depiction of heat transfer processes. We can also observe that the velocity of heat transfer fluid changes sufficiently with changing the diameter of channels.

Finally, in Table 1, we summarized the estimated relaxation times for the cells studied with different diameters of microwires. As evident from the table, the relaxation time increases almost linearly with increasing microwire diameter.

Knowledge of time relaxation may be useful to estimate approximately the maximum frequency of a magnetic cooling device in which microwires are considered as a working body. Thus, the working frequency can be expressed as $2 f=1 / t_{\text {rel }}$, where $t_{\text {rel }}$ is the relaxation time, factor 2 is the cooling and the heating cycles. We find that the maximum frequency of

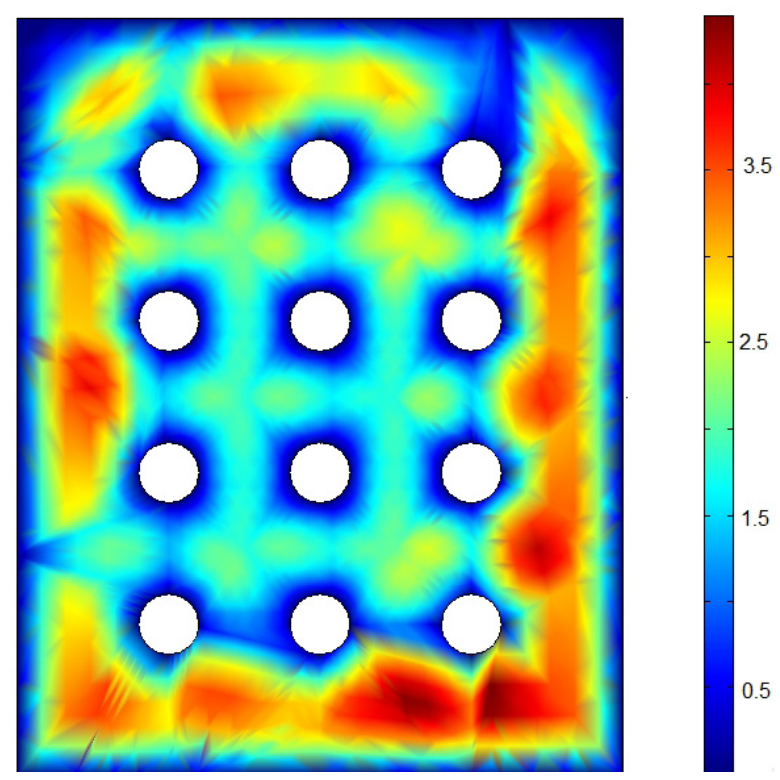

Table 1. Calculated relaxation time for both C-1 and C-2 cells with a different diameter of microwires.

\begin{tabular}{|c|c|c|c|c|}
\hline \multirow{2}{*}{ Diameter $(\mu \mathrm{m})$} & \multicolumn{2}{|c|}{$\mathrm{C}-1$} & \multicolumn{2}{c|}{$\mathrm{C}-2$} \\
\cline { 2 - 5 } & $t_{\text {rel }}(\mathrm{ms})$ & $f(\mathrm{~Hz})$ & $t_{\text {rel }}(\mathrm{ms})$ & $f(\mathrm{~Hz})$ \\
\hline 10 & 0.9 & 555 & 0.8 & 625 \\
\hline 20 & 1.1 & 455 & 1.1 & 455 \\
\hline 30 & 1.7 & 294 & 1.6 & 313 \\
\hline 40 & 2.2 & 227 & 1.9 & 263 \\
\hline 50 & 2.4 & 208 & 2.2 & 227 \\
\hline
\end{tabular}

the cooling device with C-2 cell is higher, suggesting that the microwire arrangement plays a crucial role in the heat transfer processes with a fixed set of initial parameters.

\section{Conclusion}

In this paper, we investigated the heat transfer processes in a three-dimensional magnetic cooling cell containing 12 Heusler-type $\mathrm{Ni}_{45} \mathrm{Co}_{5} \mathrm{Mn}_{37} \mathrm{In}_{13}$ microwires. The diameter of microwires ranged from 10 to $50 \mu \mathrm{m}$, while the length of microwires was fixed to $1 \mathrm{~mm}$. The calculations were performed for two cells with squarely and hexagonally packed layers of microwires. The volume of the cooling cell and the cell parameters were similar for both cells. It was found that in the case of a microwire with a diameter of $50 \mu \mathrm{m}$, the relaxation time was 2.4 and $2.2 \mathrm{~ms}$ for C- 1 and C-2 cell, respectively. The lower relaxation time indicates that heat transfer can occur faster in the C-2 cell with the same initial coolant velocity and equal wire thickness. It was also established that the transfer of thermal energy occured more efficiently and faster in the cell C-2. From the presented data, we conclude that the hexagonally packed arrangement of microwires is optimal and effective and can be promising for the magnetic cooling technology.

Acknowledgments. This study was supported by the Russian Science Foundation, project no. 17-72-20022.

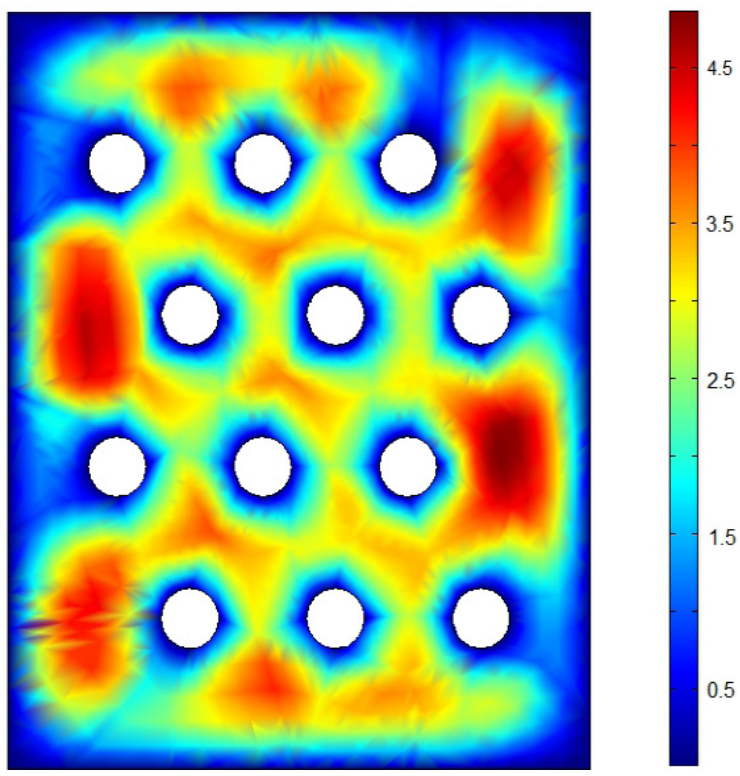

$\mathrm{b}$

Fig. 5. (Color online) The contour maps of velocity fields for C-1 (a) and C-2 (b) cells involving microwires with diameter $40 \mu \mathrm{m}$. 


\section{References}

1. A. Kitanovski, W.E. Peter. Int. J. Refrig. 29, 3 (2006). Crossref

2. K.A. Gschneidner, V.K. Pecharsky, A. O. Tsokol. Rep. Prog. Phys. 68, 1479 (2005). Crossref

3. J. Liu, T. Gottschall, K. Skokov, J. Moore, O. Gutfleisch. Nature Mater. 11, 620 (2012). Crossref

4. O. Pavlukhina, V. Buchelnikov, V. Sokolovskiy, M. Zagrebin. Solid State Phenomena. 190, 347 (2012). Crossref

5. R. Das, S. Sarma, A. Perumal, A. Srinivasan. J. Appl. Phys. 109, 07A901 (2011). Crossref

6. O. Pavlukhina, V. Buchelnikov, V. Sokolovskiy. Functional Materials. 19, 97 (2012).

7. O. Pavlukhina, V. Sokolovskiy, V. Buchelnikov. Solid State Phenomena. 233-234, 251 (2015). Crossref

8. D. Y. Cong, S. Roth, L. Schultz. Acta Mater. 60, 5335 (2012). Crossref

9. S. Fabbrici et al. Entropy. 16, 2204 (2014). Crossref

10. O. Pavlukhina, V. Buchelnikov, V. Sokolovskiy. Materials Science Forum. 845, 138 (2016). Crossref

11. O. Pavlukhina, V. Buchelnikov, V. Sokolovskiy. MATEC Web of Conferences. 33, 02002 (2015). Crossref

12. C. Felser, A. Hirohata. Heusler Alloys: Properties, Growth, Applications. Springer, Cham (2015) 486 p. Crossref

13. T. Gottschall, K. Skokov, B. Frincu, O. Gutfleisch. Appl. Phys. Lett. 106, 121901 (2015). Crossref

14. A. Tura, A. Rowe. Int. J. Refrig. 34, 628 (2011). Crossref

15. D.S. Arnold, A. Tura, A. Ruebsaat-Trott, A. Rowe. 5th International Conference on Magnetic Refrigeration at Room Temperature. Grenoble, France (2012) p. 309.

16. S. Jacobs, J. Auringer, A. Boeder, et al. 5th International Conference on Magnetic Refrigeration at Room Temperature. Grenoble, France (2012) p. 421.

17. M. Balli, O. Sari, C. Mahmed, et al. Appl. Energy. 98, 556 (2012). Crossref
18. C.R. H. Bahl, D. Velazquez, K.K. Nielsen, et al. Appl. Phys. Lett. 100, 121905 (2012). Crossref

19. P. V. Trevizoli, J.R. Barbosa Jr., R.T. S. Ferreira. Int. J. Refrig. 34, 1518 (2011). Crossref

20. V.V. Khovaylo, V.V. Rodionova, S.N. Shevyrtalov, V. Novosad. Phys. Status Solidi B. 251, 2104 (2014). Crossref

21. V. Zhukova, M. Ipatov, A. Granovsky, A. Zhukov. J. Appl. Phys. 115, 17A939 (2014). Crossref

22. A. Sarlah, J. Tusek, A. Poredos. J. Mech. Eng. 58, 16 (2012). Crossref

23. L. Kuhn, N. Pryds, C. Bahl, A. Smith. J. Phys: Conf. Ser. 303, 012082 (2011). Crossref

24. K. Nielsen et al. Int. J. Refrig. 34, 603 (2011). Crossref

25. K. K. Nielsen, C. R. H. Bahl, A. Smith, et al. Int. J. Refrig. 32, 1478 (2009). Crossref

26. K. Engelbrecht, J. Tusek, K. K. Nielsen, et al. J. Phys. D: Appl. Phys. 46, 255002 (2013). Crossref

27. M. Vazquez, H. Chiriac, A. Zhukov, et al. Phys. Status Solidi A. 208, 493 (2011). Crossref

28. M.I. Ilyn, V. Zhukova, J.D. Santos, et al. Phys. Status Solidi A. 205, 1378 (2008). Crossref

29. A. Zhukov, V. Rodionova, M. Ilyn, et al. J. Alloys Compd. 575, 73 (2013). Crossref

30. V. Zhukova, A. M. Aliev, R. Varga, et al. J. Supercond. Nov. Magn. 26, 1415 (2013). Crossref

31. O. Pavlukhina, V. Sokolovskiy, V. Buchelnikov. 7th International Conference on Magnetic Refrigeration at Room Temperature (Thermag VII). Proceedings. Turin, Italy (2016) p 174. Crossref

32. O. Pavlukhina, V. Sokolovskiy, V. Buchelnikov. Phys. Status Solidi A. 213 (2), 390 (2016). $\underline{\text { Crossref }}$

33. O.C. Zienkiewicz, K. Morgan. Finite Elements and Approximations. John Wiley \& Sons, New York (1983) $328 \mathrm{p}$.

34. O. C. Zienkiewicz, R. L. Taylor. Fluid Dyn. 3, 347 (2000). 